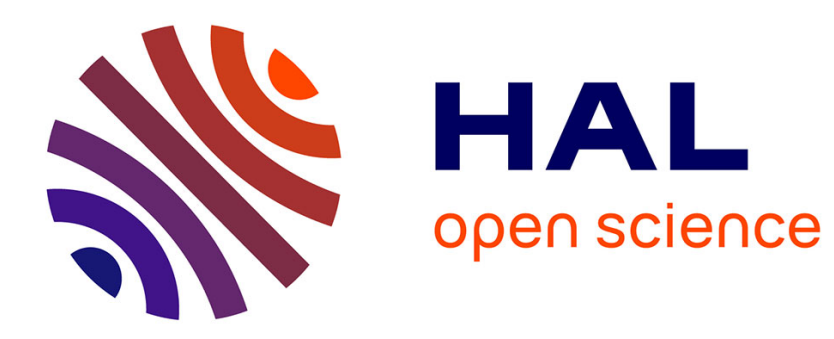

\title{
Tunable architecture for flexible and highly conductive graphene-polymer composites
}

\author{
Amélie Noël, Jenny Faucheu, Mathilde Rieu, Jean-Paul Viricelle, Elodie \\ Bourgeat-Lami
}

\section{- To cite this version:}

Amélie Noël, Jenny Faucheu, Mathilde Rieu, Jean-Paul Viricelle, Elodie Bourgeat-Lami. Tunable architecture for flexible and highly conductive graphene-polymer composites. Composites Science and Technology, 2014, 95, pp.82-88. 10.1016/j.compscitech.2014.02.013 . hal-00966122

\section{HAL Id: hal-00966122 \\ https://hal.science/hal-00966122}

Submitted on 27 Mar 2014

HAL is a multi-disciplinary open access archive for the deposit and dissemination of scientific research documents, whether they are published or not. The documents may come from teaching and research institutions in France or abroad, or from public or private research centers.
L'archive ouverte pluridisciplinaire HAL, est destinée au dépôt et à la diffusion de documents scientifiques de niveau recherche, publiés ou non, émanant des établissements d'enseignement et de recherche français ou étrangers, des laboratoires publics ou privés. 


\title{
Tunable architecture for flexible and highly conductive graphene-polymer composites
}

\author{
Amélie Noël ${ }^{1}$, Jenny Faucheu ${ }^{1, *}$, Mathilde Rieu ${ }^{2}$, Jean-Paul Viricelle ${ }^{2}$ and Elodie Bourgeat- \\ Lami $^{3}$
}

${ }^{1}$ Ecole Nationale des Mines, SMS-EMSE, MPI, CNRS : UMR 5307, LGF, 158 Cours Fauriel, 42023 Saint Etienne, Cedex 02, France

*faucheu@emse.fr

${ }^{1}$ Ecole Nationale des Mines, SPIN-EMSE, PRESSIC, CNRS : UMR 5307, LGF, 158 Cours Fauriel, 42023 Saint Etienne, Cedex 02, France

${ }^{3}$ Université de Lyon, Université Lyon 1, CPE Lyon, CNRS : UMR 5265, Laboratoire de Chimie, Catalyse, Polymères et Procédés (C2P2), LCPP Group, 43 Boulevard du 11 Novembre 1918, 69616 Villeurbanne Cedex, France

\begin{abstract}
Printed electronics, particularly on flexible and textile substrates, raised a strong interest during the past decades. This work presents a good candidate for conductive inks based on a graphene/polymer nanocomposite material that gathers three main benefits that are 1- neither clogging nor flocculation, 2- spontaneous film formation around room temperature, 3- high conductivity. Nanosized Multilayered Graphene (NMG) is produced through a solvent-free procedure, using a grinding process in water. These NMG suspensions are used to elaborate conductive composite materials through physical blending with emulsifier-free latex. The nanocomposite microstructure exhibits a well-defined cellular architecture that highlights the formation of continuous paths of fillers throughout the material. The conductivity behavior of the nanocomposite material was efficiently described using a percolation model: the conductivity can be tuned by changing the NMG content and the latex size. A low percolation threshold $(0.1$ vol $\%)$ was obtained and the electrical conductivity
\end{abstract}


reached 217 S.m ${ }^{-1}$ for 6 vol\% NMG. Efficient film forming occurs at room temperature leading to continuous and deformable materials, which is adequate for printing on flexible and textile substrates. The applicability in electronics is demonstrated by the use of the nanocomposite material in replacement of copper wires in a LED setup.

Keywords: A. Nanocomposites, A. Flexible composites, B. Electrical properties

\section{1- Introduction}

In the past decades, flexible and textile substrates raised a strong interest in printed electronics applications. Wearable electronics would transform traditional textile and apparel products into lightweight, wireless and wearable intelligent devices [1]. Currently, conductive inks, in particular ink-jet inks, are mostly based on dispersions of metallic particles in an aqueous or organic medium [2]. To reach the adequate particle sintering, an annealing step at high temperature is required, around $500-1000{ }^{\circ} \mathrm{C}$ [3]. Moreover, the resulting sintered material can undergo only very small deformations. For those two reasons, flexible and textile substrates cannot be used with these inks.

The aim of this paper is to present a simple processing route for producing conductive inks that form a continuous film at room temperature and that exhibit high deformability after drying. The strategy is based on architectured nanocomposites allying conductivity characteristics of Nanosize Multilayered Graphene (NMG) and deformability of a polymer binder. The nanocomposite architecture is tuned using a latex route [4]. The production of conductive nanocomposites by blending a polymer latex with graphene-based particles has been already presented by several authors [5], [6] and [7]. However in all of these works, Graphene Oxide (GO) and reduced Graphene Oxide (rGO) was used, increasing the number of processing steps and chemical products needed. Moreover, in most of these works, the composite blends were not stable: the composite solids ended up flocculating and a subsequent hot-pressing step was needed to produce a continuous composite material with 
acceptable mechanical and electrical properties. For nanocomposite blend formulation, the latex route exhibits two major advantages as compared to the melt route or solution route. First, this synthetic route is sustainable as a latex is made of polymer nanospheres in an aqueous suspension without using organic solvent. Second, the latex route favors the built-up of a tunable architecture of fillers. This specific architecture, in turn, favors the formation of a percolating network of fillers at lower filler content [8]. As a result, the final nanocomposite microstructure counts two interpenetrated networks, one made of the polymer matrix and the other one made of percolating fillers [9],[10]. The architecture of the polymer matrix is constrained by the spherical shape of the latex particles and the shape of the fillers can influence the percolation threshold. The use of 2D-fillers (platelets) would both favor filler contacts compared to 1D-fillers (nanotubes) and lower the percolation threshold compared to 3D-fillers (spheres). Thus, for geometrical considerations, platelet-like fillers [11] seems to be a good choice to build up an efficient network with polymer nanospheres.

The chosen size ratio between the polymer nanospheres and the fillers is a compromise. On the one hand, the platelets have to be large enough to limit the number of conductive platelets needed to cover the surface of the latex spheres and so the number of contacts. Indeed, intuitively, the contacts between fillers will have a lower conductivity than the intrinsic conductivity of the filler itself. On the other hand, the platelets have to be small enough in order not to destabilize the blend or hinder the film formation process of the latex nanospheres. Hereafter, the fabrication process and characterization of home-made conductive graphene-based nanoplatelets (NMG) are detailed. Secondly, the highly conductive behavior of NMG-based nanocomposites obtained through latex blends are discussed in terms of filler architecture through a percolation approach. Finally, it is demonstrated by mounting a simple electronic setup that this procedure provides a good candidate for conductive ink for printed electronics on flexible and textile substrates. 


\section{2- Experimental}

2.1- Materials: Graphite flakes (7-10 $\mu \mathrm{m}$ with 99\% purity) from Alfa Aesar and Sodium dodecyl benzene sulfonate (SDBS, Aldrich) were used as received. Potassium persulfate (KPS), methyl methacrylate and $n$-butyl acrylate monomers purchased from Aldrich were used without further purification.

2.2- Preparation of Nanosize Multilayered Graphene (NMG): The preparation of the conductive particles, NMG, was performed using a bead milling procedure in wet media. Prior to the milling procedure, the graphite flakes ( $40 \mathrm{~g}$ ) and the surfactant (SDBS, $2 \mathrm{~g}$ ) were dispersed in water $(400 \mathrm{~mL})$ under agitation during 12 hours. The surfactant favored the grinding process and the stability of both the graphite flakes and the NMG further produced. The grinding experiments were carried out in a horizontal laboratory attritor (Minizeta, Netzsch Feinmahltechnik GmbH, Selb, Germany) during 4 hours at 1500 rpm, using Yttriumstabilized zirconium oxide grinding beads (ZetaBeads Plus, $0.4 \mathrm{~mm}$, Netzsch) as grinding media. A sonicator was added in the attritor loop to improve graphite exfoliation and scission. A stable NMG suspension was isolated through decantation of the larger graphite flakes. It was used as-is or after increase of NMG-content by water evaporation.

\section{3- Preparation of surfactant-free acrylate copolymer latexes via Free radical Emulsion} Polymerization: The polymer matrix used is a poly (methyl methacrylate-co-butyl acrylate) $\mathrm{P}(\mathrm{MMA}$-co-BA) latex spheres in a water suspension were synthesized using free-radical emulsion polymerization. The monomers (methyl methacrylate $(12.5 \mathrm{~g})$ and butyl acrylate $(12.5 \mathrm{~g})$ were poured in the reactor for degassing under nitrogen. Water (95 g) was added for further degassing and the reactor was pre-heated at $70{ }^{\circ} \mathrm{C}$. After injection of the initiator (KPS $(0.25 \mathrm{~g}))$ dissolved in water $(5.0 \mathrm{~g})$, the polymerization was carried out during 5 hours at 70 ${ }^{\circ} \mathrm{C}$. A final monomer conversion of $99 \%$ was reached. The average particle size determined by DLS was $650 \mathrm{~nm}(0.3$ Polydispersity Index). 
2.4- Preparation of the nanocomposite films: Prior to latex blending, the NMG platelets were introduced into the latex suspension drop-by-drop to increase the adsorption of NMG onto the latex nanospheres. The latex $(1.0 \mathrm{~g})$ was poured in a beaker and placed in an ultrasonic bath. The adequate amount of NMG suspension was placed in a syringe. The NMG-content and the volume of suspension depended on NMG-content of the final composite that was aimed. Finally, the composite latex blends were poured into silicone mold and dried at $40{ }^{\circ} \mathrm{C}$.

2.5- Characterization: Microstructural characterizations were performed using complementary techniques. Dynamic Light Scattering (DLS, Vasco) was performed on diluted suspensions. A drop of the NMG suspension was deposited on carbon-coated 300mesh copper grid and dried prior to Transmission Electron Microscopy observations (TEM Philips CM120) and High Resolution TEM (HR-TEM JEOL 2100 F) ) at the CT $\mu$ (Centre Technologique des Microstructures, Lyon 1 University). The NMG suspension was spincoated on Silicon wafers and washed using deionized water prior to Atomic Force Microscopy (AFM, JPK Nanowizard 3) characterizations. The washing step was performed to eliminate surfactant traces on the surface that might influence the AFM measurements. Thin foil TEM-specimens $(<100 \mathrm{~nm})$ of the nanocomposite material were prepared using a diamond knife on a cryo-ultramicrotome equipment.

The electrical conductivity was measured using a four-probe setup (with gold contacts and $3.48 \mathrm{~mm}$ of distance between each probe) equipped with a conductimeter (Keithley 2400) [12].

\section{3- Results and Discussion}

\section{1- Processing steps of NMG/latex nanocomposites}

In this work, the challenge relies on the production of adequate conductive fillers with specific dimensional characteristics that do not destabilize the latex during blending. In the literature the most popular route to synthesize NMG is based on the so-called Hummer's 
method [13]. Despite its popularity, this method presents noticeable disadvantages as it relies on a long and multistep synthesis using many chemical products. In Hummer's method, GO is produced from graphite flakes and reduced into rGO. After this reduction step, some functional groups containing oxygen remains on the rGO surface [14] that might affect both the interactions with the latex particles and the final electrical properties of the composite. As an alternative, Knieke et al. proposed a production of NMG based on the mechanical delamination of graphite in wet grinding media [15]. This mechanical method is not popular, yet it is cost-effective and avoids organic solvents. In our work, this procedure was chosen and the NMG water suspensions were conveniently used as-is in the subsequent processing steps of the nanocomposite material. The delamination process does require the use of a large amount of graphite flakes, but these are cheap and reusable raw materials. After 4 hours of wet grinding, $0.8 \mathrm{~g}$ of NMG in water suspension is obtained from $40 \mathrm{~g}$ of graphite flakes. The graphite suspensions are stabilized with addition of SDBS. This surfactant contains a benzene group that likely improves the NMG stabilization through $\pi-\pi$ interactions, as compared to the most commonly used surfactant Sodium Dodecyl Sulfate (SDS) [16]. After the grinding step, the suspensions were settled overnight in order to remove the remaining graphite flakes through sedimentation. The concentration of the produced NMG suspensions was $2 \mathrm{mg} \mathrm{mL} \mathrm{L}^{-1}$ which is two times higher than common concentrations of graphene oxide suspensions obtained through Hummer's method [17].

To assess the NMG thickness $h$ AFM measurements were carried out (Figure 1a). The NMG suspension was spin-coated on a silicon wafer and washed with deionized water in order to remove surfactant traces. This washing step might remove some of the NMG particles from the wafer [15]. The cumulative number distribution, $N(h)$, given in Figure 1c, describes the amount of NMG sheets that have a thickness equal or smaller than a given thickness $h$ and $N(h)$ was assessed from $150 \mathrm{NMG}$ sheets from various areas of the silicon wafer. The $50 \%$ 
percentile $h_{50}$ is $3 \mathrm{~nm}$ and the $90 \%$ percentile $h_{90}$ is around $3.5 \mathrm{~nm}$, i.e. $90 \%$ of created NMG sheets are thinner than $4 \mathrm{~nm}$. In addition, the sheet-like morphology is illustrated in Figure $1 \mathrm{~b}$ showing profile of three different NMG particles.

To assess the NMG lateral size $d$, AFM and TEM observations were combined DLS measurements in order to ensure a more complete description of the NMG morphology. On the one hand, AFM gives the exact characterization of NMG morphology but this analysis remains very local. The lateral size over 500 NMG particles was assessed through AFM image analysis considering a disk-like equivalent particle. On the other hand, DLS measurements provide a statistical description of the size distribution; however, uncertainties might be introduced as the particles in suspension are considered spherical. Cumulative distributions of the lateral size assessed through AFM and DLS are plotted in Figure 2. Slight differences in general shape are noted but both distributions are consistent in that the 50\% percentile $d_{50}$ are between 30 and $60 \mathrm{~nm}$. The average lateral size of the NMG nanoplatelets produced was about ten times smaller than the latex diameter $(650 \mathrm{~nm})$, described hereafter.

On the TEM image shown on Figure 3a, two large NMG platelets (around $150 \mathrm{~nm}$ lateral size) are visible (arrows). The different grey shades are consistent different NMG thicknesses: this can come from NMG overlap or NMG folding. The HR-TEM image on Figure $3 b$ shows a NMG platelet that counts 7 graphene layers.

In addition to adequate NMG size characteristics for latex blending, these NMG water suspensions exhibited good self-stability (no sedimentation nor flocculation after 2 weeks). The suspensions were used as-is or after concentrated suspensions after water evaporation to produce NMG/polymer conductive inks through latex blending. The copolymer P(MMA-coBA) has a glass transition temperature around $15^{\circ} \mathrm{C}$. The latex was prepared through free radical polymerization without emulsifier. The latex nanospheres had an average diameter of $650 \mathrm{~nm}$ that was assessed through DLS measurements. 
The NMG suspensions of different solids contents were slowly added to the P(MMA-co-BA) latex in an ultrasonic bath and left standing for $10 \mathrm{~min}$ (Figure 4). The blends exhibited a homogeneous dark grey color and no sedimentation, phase separation nor flocculation was observed. They were poured into silicone molds and left to dry overnight at $40{ }^{\circ} \mathrm{C}$. The latex film forming process is commonly described as a multistep process. As water evaporates, the latex nanospheres come into contact in a compact arrangement, and then deform to fill in the porosity and finally the polymer chains diffuse from one nanosphere to the other. Despite the presence of nanoplatelets in the water phase, flexible free-standing films (around $200 \mu \mathrm{m}$ thick) were easily extracted from the silicon molds. Eight composite films (specimens 1 to 8) were produced and the NMG contents in the films are detailed in Table 1. A blank polymer film was also produced as a reference (specimen 0$)$.

Note that after the film forming process, surfactant molecules likely exude at the air-film and mold-film interfaces which might influence the conductivity measurements [18]. Thus, all the composites films were washed several times in deionized water prior to further characterization.

\section{2- Morphological characterization of NMG/latex nanocomposites}

Thin foil TEM-specimens were prepared to assess the nanostructure of the final composite films (Figure 5 and 6). On the TEM micrographs, light grey domains are holes in the thin cross section. The polymer matrix appears in medium grey as a homogeneous background. Due to their very small thickness, the NMG platelets are visible when they are edge on, then they appear as dark sticks or dark aggregates. Compact arrangement of latex particles surrounded with NMG platelets are visible as domains distributed as a cellular structure. These TEM observations are consistent with a geometrical description of the composite as a close-packed cubic arrangement of deformed spheres. 
At lower magnification, NMG paths are visible throughout the nanocomposite material. The TEM micrograph shows a 2D-cross-section of the 3D-NMG network that is developed throughout the whole material. These cross-sections give insights of the network density. As expected, with an increase of NMG content from $0.5 \%$ vol to $5 \%$ vol, the network density is clearly higher: an increasing number of paths are visible and an overall cellular architecture appears (Figure 6).

Thanks to the latex blending route, using fillers with adequate sizes, flexible NMG/polymer composite materials with an architectured microstructure were obtained. The influence of NMG content on the nanocomposite conductivity characteristics is explored hereafter.

\section{3- Conductivity characteristics of NMG/latex nanocomposites}

The electrical conductivity of the nanocomposite materials was measured using a four probe conductimeter. Ten measurements were performed on each side of the free-standing composite film. Figure 7a shows the average electrical conductivity as a function of the NMG content. Note that the conductivity of the blank sample $\left(x_{N M G}=0\right)$ was not measured but set to a value for common dielectric materials $\left(10^{-9} \mathrm{~S}^{\left.-\mathrm{m}^{-1}\right)}\right.$ [19]. The plot exhibits a steep increase at low NMG content and a slower increase for a NMG volume fraction above $2 \times 10^{-2}$. A maximum value of $217 \mathrm{~S} . \mathrm{m}^{-1}$ was reached at $6.1 \times 10^{-2} \mathrm{NMG}$ volume fraction. To the best of our knowledge, this conductivity value is among the highest value available in the literature for graphene-latex composites [20]. To highlight the influence of the NMG 3D-network on conductivity, the behavior of graphene-based nanocomposites is usually described through a probabilistic approach called percolation theory [21]. In this approach, the probability of forming a percolating path of fillers, i.e. a path of fillers that crosses the whole sample, is described in terms of filler volume fraction and geometrical filler arrangement. In particular, the theoretical or statistical percolation threshold is defined as the minimum volume fraction 
of fillers needed to form the first percolating path. Experimentally, the addition of conductive fillers to a dielectric polymer can result in a conductive composite if enough paths are formed to enable electron transport. Intuitively, the minimum filler content needed, called experimental percolation threshold hereafter, is equal or higher than the value derived from the statistical description of the percolation threshold and depends on the sensitivity of the conductimeter equipment. In this work, the experimental percolation threshold was evaluated as shown in Figure 7a. As dielectric materials are commonly described as materials with conductivity lower than $10^{-8} \mathrm{~S}^{-\mathrm{m}^{-1}}$, the experimental percolation threshold lies between $1 \times 10^{-3}$ and $2 \times 10^{-3}$. This value is also consistent with the steep increase observed for the conductivity in Figure 7.

Based on the percolation theory, Kirkpatrick proposed an equation to define the conductivity of biphasic materials comprising a conductive phase and a dielectric phase [22]:

$\sigma_{c}=\sigma_{0}\left[\frac{x_{N M G}-x_{c}}{1-x_{c}}\right]^{t}$

where $\sigma_{c}$ is the conductivity of the nanocomposite material, $x_{N M G}$ is the filler volume fraction, $x_{c}$ is the percolation threshold, $\sigma_{0}$ is the filler's conductivity in the composite film and $t$ is a critical exponent [23]. Note that this description is only valid for $x_{N M G}>x_{c}$. In literature, the statistical percolation threshold is said to depend on the lattice dimensionality ( 3 dimensions in our case) and lattice geometry (more likely close to faced-centered cubic in these latexbased materials) [24]. The critical exponent $t$ is a universal exponent that is said to mainly depend on the dimensionality of the lattice. Using Equation (1), a linear trend is obtained for the $\log$ - $\log$ plot showing $\log \left(x_{N M G^{-}} x_{c}\right)$ on the x-axis and $\log \left(\sigma_{c}\right)$ on the y-axis, with $x_{c}=$ $1.0 \times 10^{-3}, \sigma_{0}=10^{4} \mathrm{~S} . \mathrm{m}^{-1}$, and $t=1.72$ as illustrated in Figure 7 (inset). All three parameters $\left(x_{c}\right.$, $\sigma_{0}$ and $t$ ) are physically consistent. First, the percolation threshold for $x_{c}$ is consistent with the preliminary graphical evaluation mentioned earlier. The critical exponent for a statistical description of a 3D percolation network is documented in literature around 1.6-2.0 [25], [26]. 
The filler's conductivity $\sigma_{0}$ describes the conductivity of graphene sheets assembled in the nanocomposite. Thus, both the intrinsic conductivity of the filler and the conductivity of the filler-filler contact have to be taken into account. Values for the intrinsic conductivity of graphene monolayers are largely documented in literature around $10^{7}-10^{8} \mathrm{~S}^{-1} \mathrm{~m}^{-1}$ [27]. However, these extremely high values do not take into account the filler-filler contacts. Marinho et al. conducted an experimental study on conductivity of powder compacts made of graphite, graphene and Carbon Nanotube (CNT) powders [28]. These authors measured $2.12 \times 10^{3} \mathrm{~S} . \mathrm{m}^{-1}$ for the graphite compacts, $2.62 \times 10^{2} \mathrm{~S} . \mathrm{m}^{-1}$ for graphene compacts and $5.43 \times 10^{2}$ S.m ${ }^{-1}$ for CNT compacts. The highest conductivity exhibited by the graphite compacts might be due to larger particle-particle contacts that overcome the lower intrinsic conductivity of graphite as compared to graphene and CNT. Based on these results, $\sigma_{0}=10^{4}$ $\mathrm{S}^{-1} \mathrm{~m}^{-1}$ is a consistent order of magnitude for the conductivity of NMG networks. Finally, the conductivity behavior observed for these nanocomposite materials is consistent with a threedimensional percolation behavior.

In order to demonstrate the potential application of our conductive films, a basic electronic setup based on a light emitting diode (LED) was mounted (Figure 8). In this setup, small strips $\left(2 \mathrm{~mm}\right.$-wide, $20 \mathrm{~mm}$-length and $200 \mu \mathrm{m}$-thick) were cut out of sample $8\left(6.1 \times 10^{-2}\right.$ NMG volume fraction). These strips were used to replace part of the copper wires that bond the LED to the batteries. An unexpected advantage of the nanocomposite material was obtained in that in a classical LED setup a current-limiting resistor is added to protect the LED. In our proposition, a built-in resistor was obtained and the resistance value could be tuned by changing the NMG content and the dimensional characteristics of the material strip.

\section{Conclusions}

To conclude, highly conductive graphene-based composite materials have been produced through a solvent-free procedure. This processing route based on latex blends and Nanosize 
Multilayer Graphene (NMG) provides a good candidate for conductive inks for printed electronics on flexible and textile substrates. The NMG was obtained after a few hours of mechanical mild grinding of graphite micro-flakes in water suspensions. Both the NMG suspensions and the composite latex blends exhibited good shelf-stability. The compositelatex blend is based on acrylate copolymers that are already mature in the ink and paint industry: they can form continuous and deformable films without neither high temperature curing nor additional hot-pressing, which is adequate for flexible and textile substrates. Finally high conductivity values are reached $\left(217{\mathrm{~S} . \mathrm{m}^{-1}}^{-}\right.$at $6.1 \times 10^{-2} \mathrm{NMG}$ volume fraction) which is a basic requirement for electronic applications. To our knowledge, this value is among the highest value available in the literature for graphene-latex composites.

The final composite materials exhibit a micrometer-scale domain size with filler paths running throughout the material. The conductivity behavior is successfully described using a percolation approach consistent with the presence of a three dimensional filler network across the material. The potential interest for electronics was demonstrated by the use of the nanocomposite material in replacement of copper wires in a LED setup. These conductive nanocomposite suspensions could become a cheaper alternative to silver-based conductive inks for printed electronics and could open more versatile electronic applications due to the deformability of the polymer matrix, for instance, on textile substrates.

\section{Acknowledged}

The authors would like to thanks P-Y. Dugas (C2P2/CPE/CNRS) for his help concerning the TEM observations done at the CT $\mu$ (Centre Technologique des Microstructures, Université Lyon1). 


\section{References}

[1] L. Hu, M. Pasta, F.L. Mantia, L. Cui, S. Jeong, H.D. Deshazer, et al., Stretchable, Porous, and Conductive Energy Textiles, Nano Lett. 10 (2010) 708-714.

[2] M.A.H. Khondoker, S.C. Mun, J. Kim, Synthesis and characterization of conductive silver ink for electrode printing on cellulose film, Appl. Phys. A. 112 (2013) 411-418.

[3] R. Cauchois, M. Saadaoui, A. Yakoub, K. Inal, B. Dubois-Bonvalot, J.-C. Fidalgo, Impact of variable frequency microwave and rapid thermal sintering on microstructure of inkjet-printed silver nanoparticles, J. Mater. Sci. 47 (2012) 7110-7116.

[4] P.A. Steward, J. Hearn, M.C. Wilkinson, An overview of polymer latex film formation and properties, Adv. Colloid Interface Sci. 86 (2000) 195-267.

[5] C. Wu, X. Huang, G. Wang, L. Lv, G. Chen, G. Li, et al., Highly Conductive Nanocomposites with Three-Dimensional, Compactly Interconnected Graphene Networks via a Self-Assembly Process, Adv. Funct. Mater. 23 (2013) 506-513.

[6] X. Li, G. Zhang, X. Bai, X. Sun, X. Wang, E. Wang, et al., Highly conducting graphene sheets and Langmuir-Blodgett films, Nat. Nanotechnol. 3 (2008) 538-542.

[7] A. Arzac, G.P. Leal, R. Fajgar, R. Tomovska, Comparison of the Emulsion Mixing and In Situ Polymerization Techniques for Synthesis of Water-Borne Reduced Graphene Oxide/Polymer Composites: Advantages and Drawbacks, Part. Part. Syst. Charact. 31 (2014) 143-151.

[8] E. Tkalya, M. Ghislandi, A. Alekseev, C. Koning, J. Loos, Latex-based concept for the preparation of graphene-based polymer nanocomposites, J. Mater. Chem. 20 (2010) $3035-3039$.

[9] J.C. Grunlan, A.R. Mehrabi, M.V. Bannon, J.L. Bahr, Water-Based Single-WalledNanotube-Filled Polymer Composite with an Exceptionally Low Percolation Threshold, Adv. Mater. 16 (2004) 150-153. 
[10] N. Grossiord, J. Loos, C.E. Koning, Strategies for dispersing carbon nanotubes in highly viscous polymers, J. Mater. Chem. 15 (2005) 2349-2352.

[11] Z. Yang, R. Gao, N. Hu, J. Chai, Y. Cheng, L. Zhang, et al., The Prospective TwoDimensional Graphene Nanosheets: Preparation, Functionalization, and Applications, Nano-Micro Lett. 4 (2012) 1-9.

[12] S. Alwarappan, A. Erdem, C. Liu, C.-Z. Li, Probing the Electrochemical Properties of Graphene Nanosheets for Biosensing Applications, J. Phys. Chem. C. 113 (2009) 88538857.

[13] W.S. Hummers, R.E. Offeman, Preparation of Graphitic Oxide, J. Am. Chem. Soc. 80 (1958) 1339-1339.

[14] X. Gao, J. Jang, S. Nagase, Hydrazine and Thermal Reduction of Graphene Oxide: Reaction Mechanisms, Product Structures, and Reaction Design, J. Phys. Chem. C. 114 (2010) 832-842.

[15] C. Knieke, A. Berger, M. Voigt, R.N.K. Taylor, J. Röhrl, W. Peukert, Scalable production of graphene sheets by mechanical delamination, Carbon. 48 (2010) 31963204.

[16] J.H. Lee, D.W. Shin, V.G. Makotchenko, A.S. Nazarov, V.E. Fedorov, J.H. Yoo, et al., The Superior Dispersion of Easily Soluble Graphite, Small. 6 (2010) 58-62.

[17] S. Stankovich, D.A. Dikin, R.D. Piner, K.A. Kohlhaas, A. Kleinhammes, Y. Jia, et al., Synthesis of graphene-based nanosheets via chemical reduction of exfoliated graphite oxide, Carbon. 45 (2007) 1558-1565.

[18] E. Kientz, Y. Holl, Distribution of surfactants in latex films, Colloids Surf. Physicochem. Eng. Asp. 78 (1993) 255-270.

[19] J.P. Mária Omastová, Chemical preparation and characterization of conductive poly(methyl methacrylate)/polypyrrole composites, Polymer. 39 (1998) 6559-6566. 
[20] V.H. Pham, T.T. Dang, S.H. Hur, E.J. Kim, J.S. Chung, Highly conductive poly(methyl methacrylate) (PMMA)-reduced graphene oxide composite prepared by self-assembly of PMMA latex and graphene oxide through electrostatic interaction, ACS Appl. Mater. Interfaces. 4 (2012) 2630-2636.

[21] D. Stauffer, A. Aharony, Introduction to percolation theory, Taylor \& Francis, 1994.

[22] S. Kirkpatrick, Percolation and Conduction, Rev. Mod. Phys. 45 (1973) 574-588.

[23] D.S. McLachlan, C. Chiteme, C. Park, K.E. Wise, S.E. Lowther, P.T. Lillehei, et al., AC and DC percolative conductivity of single wall carbon nanotube polymer composites, J. Polym. Sci. Part B Polym. Phys. 43 (2005) 3273-3287.

[24] P.R. Sperry, B.S. Snyder, M.L. O’Dowd, P.M. Lesko, Role of Water in Particle Deformation and Compaction in Latex Film Formation, Langmuir. 10 (1994) 26192628.

[25] A.L. Efros, B.I. Shklovskii, Critical Behaviour of Conductivity and Dielectric Constant near the Metal-Non-Metal Transition Threshold, Phys. Status Solidi B. 76 (1976) 475485.

[26] J.P. Clerc, G. Giraud, J.M. Laugier, J.M. Luck, The electrical conductivity of binary disordered systems, percolation clusters, fractals and related models, Adv. Phys. 39 (1990) 191-309.

[27] W. Chen, L. Yan, P.R. Bangal, Preparation of graphene by the rapid and mild thermal reduction of graphene oxide induced by microwaves, Carbon. 48 (2010) 1146-1152.

[28] B. Marinho, M. Ghislandi, E. Tkalya, C.E. Koning, G. de With, Electrical conductivity of compacts of graphene, multi-wall carbon nanotubes, carbon black, and graphite powder, Powder Technol. 221 (2012) 351-358. 

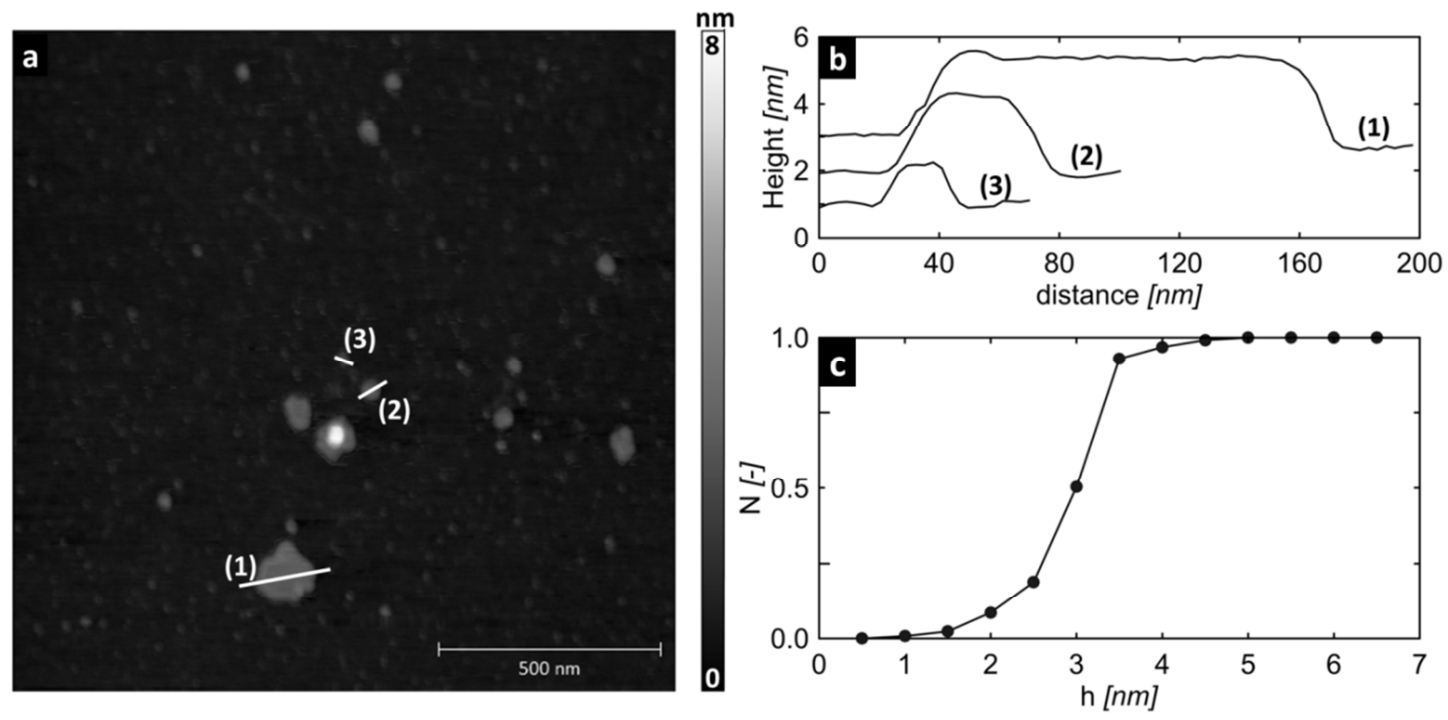

Figure 1.Thickness $h$ of the NMG nanoplatelets. (a) AFM height image, (b) Height profiles of NMG platelets extracted from AFM images (the profiles have been shifted along y-axis for clarity) and (c) Cumulative distribution of the NMG thickness.

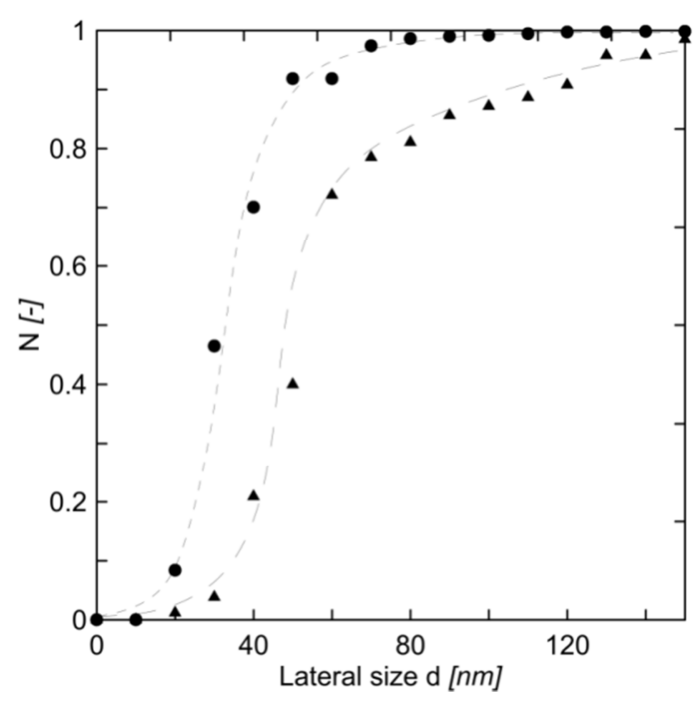

Figure 2. Cumulative distribution of the NMG lateral size $d$ assessed using AFM (triangles) and DLS (circles). 


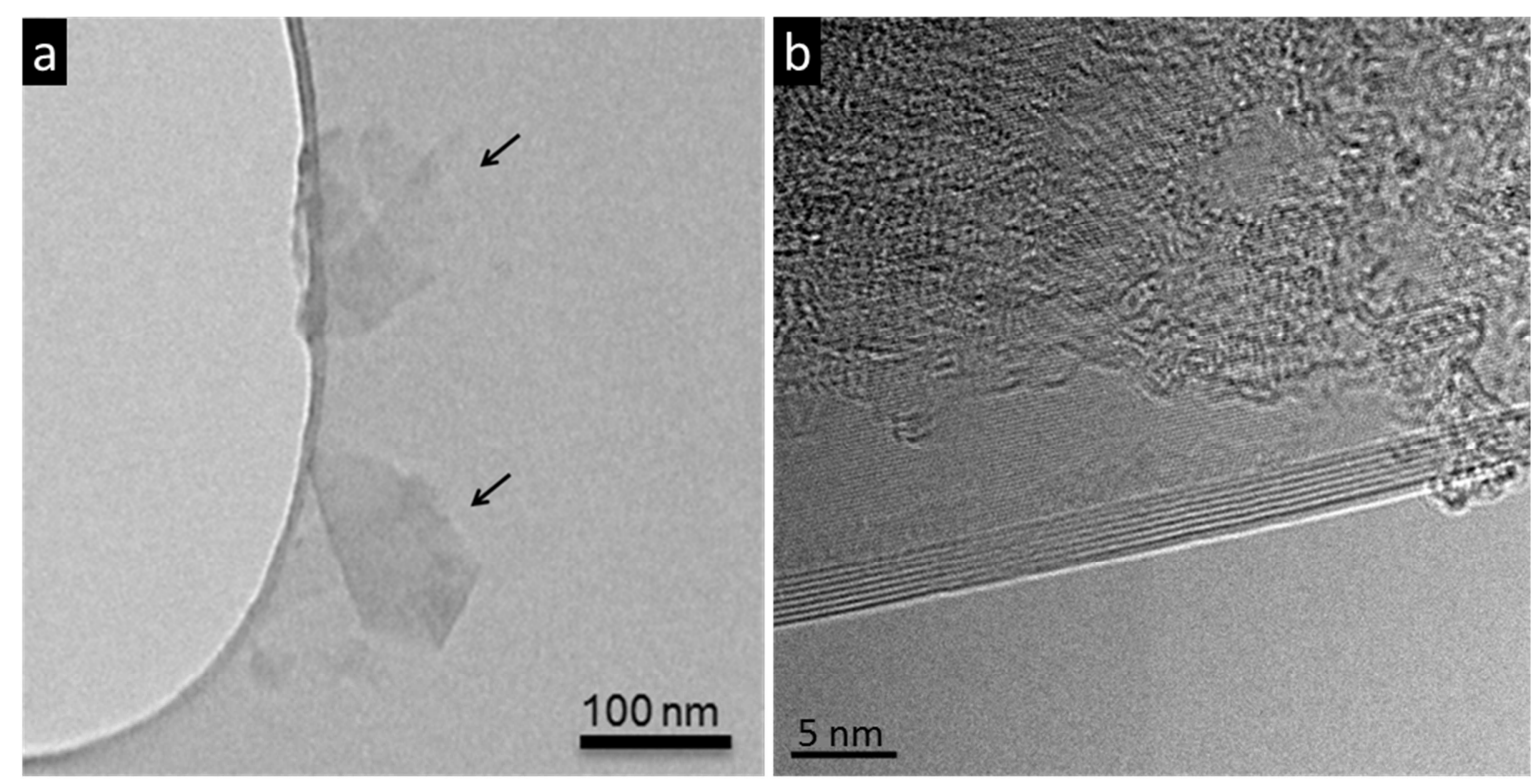

Figure 3. (a) TEM micrograph of NMG nanoplatelets (arrows), (b) HR-TEM micrograph of NMG nanoplatelet.

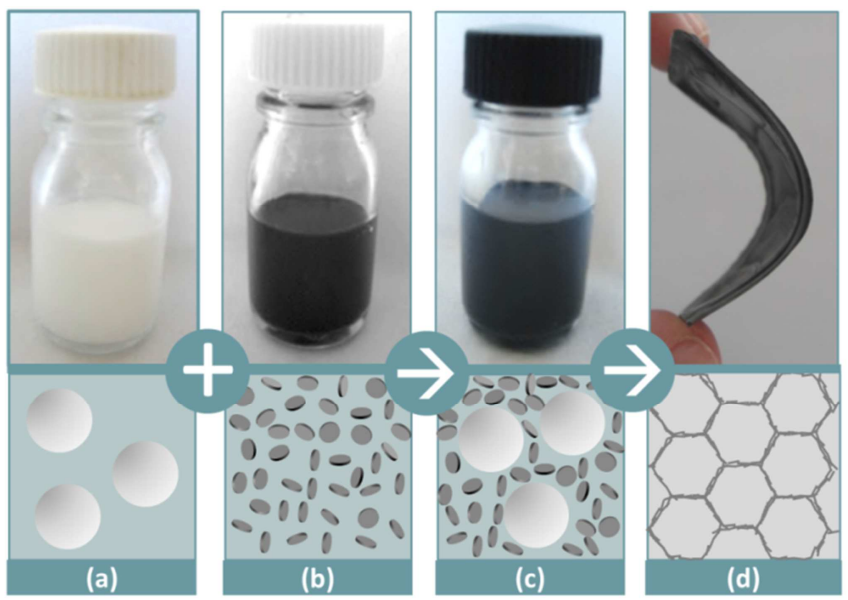

Figure 4. Processing steps of the nanocomposite materials, (a) blank latex, (b) NMG suspension, (c) NMG-latex blend, (d) flexible and conductive material obtained after water evaporation and film formation. 


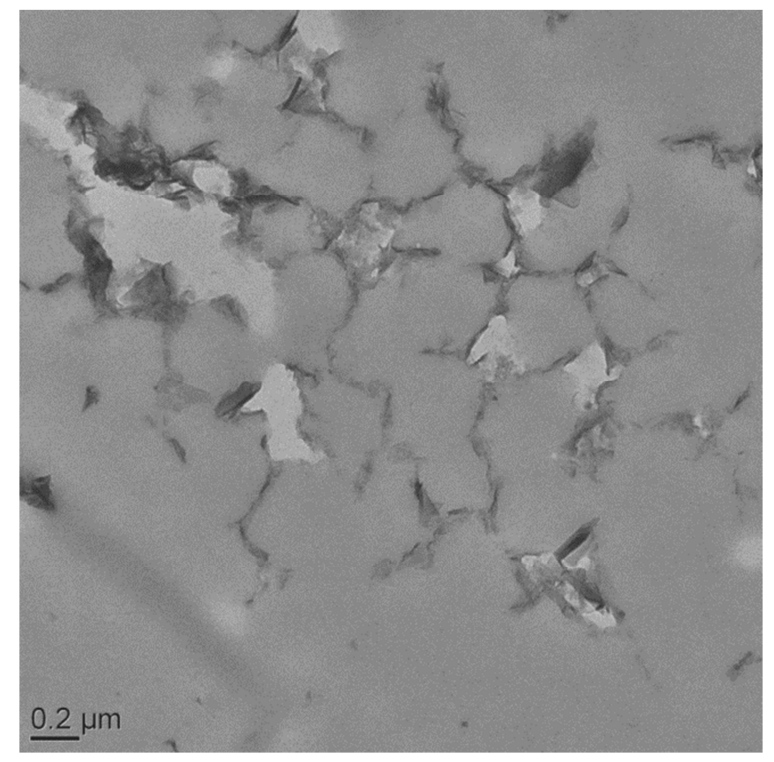

Figure 5: TEM micrograph of a thin foil extracted from sample $7\left(x_{N M G}=5 \times 10^{-2}\right)$.
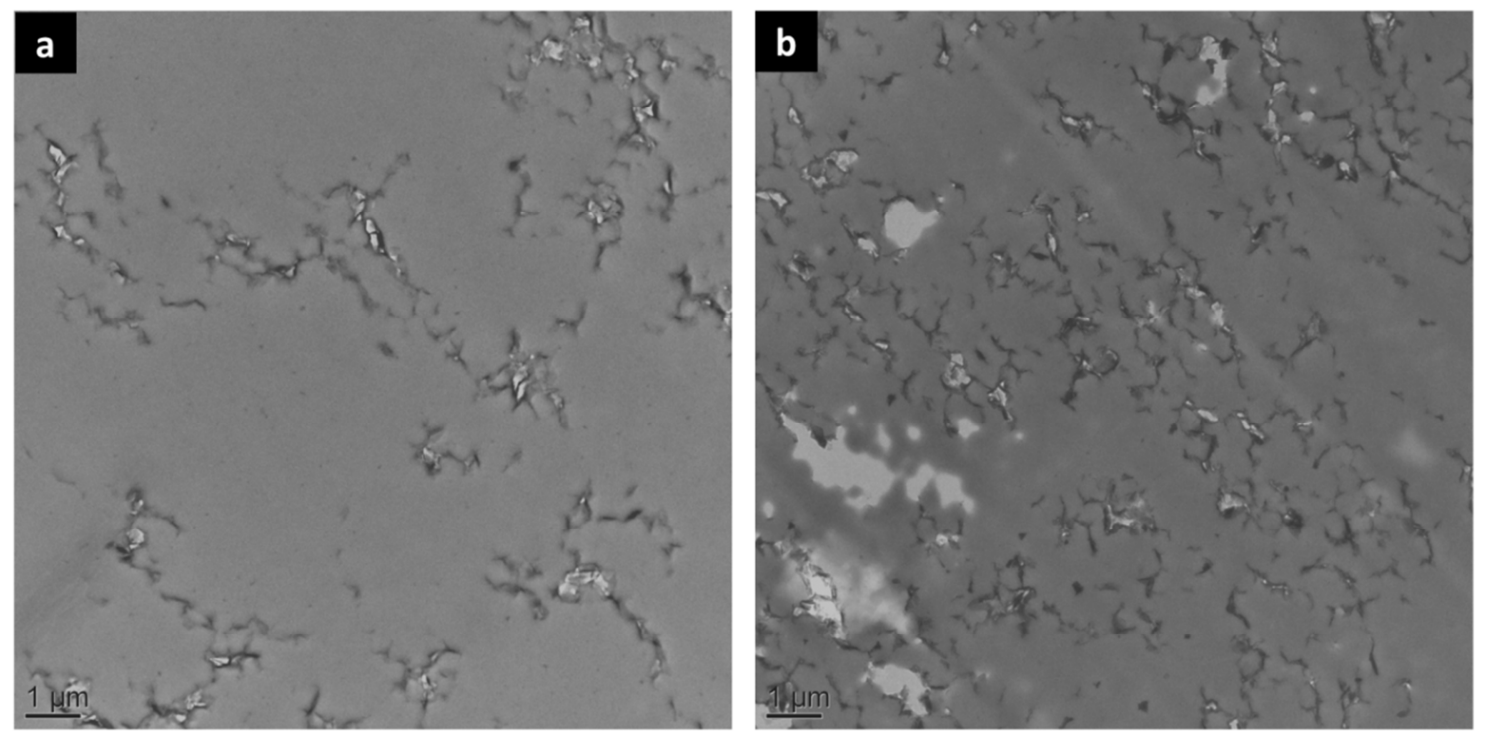

Figure 6. TEM micrograph of a thin foil extracted from (a) sample $3\left(x_{N M G}=5 \times 10^{-3}\right)$ and (b) sample $7\left(x_{N M G}=5 \times 10^{-2}\right)$ 


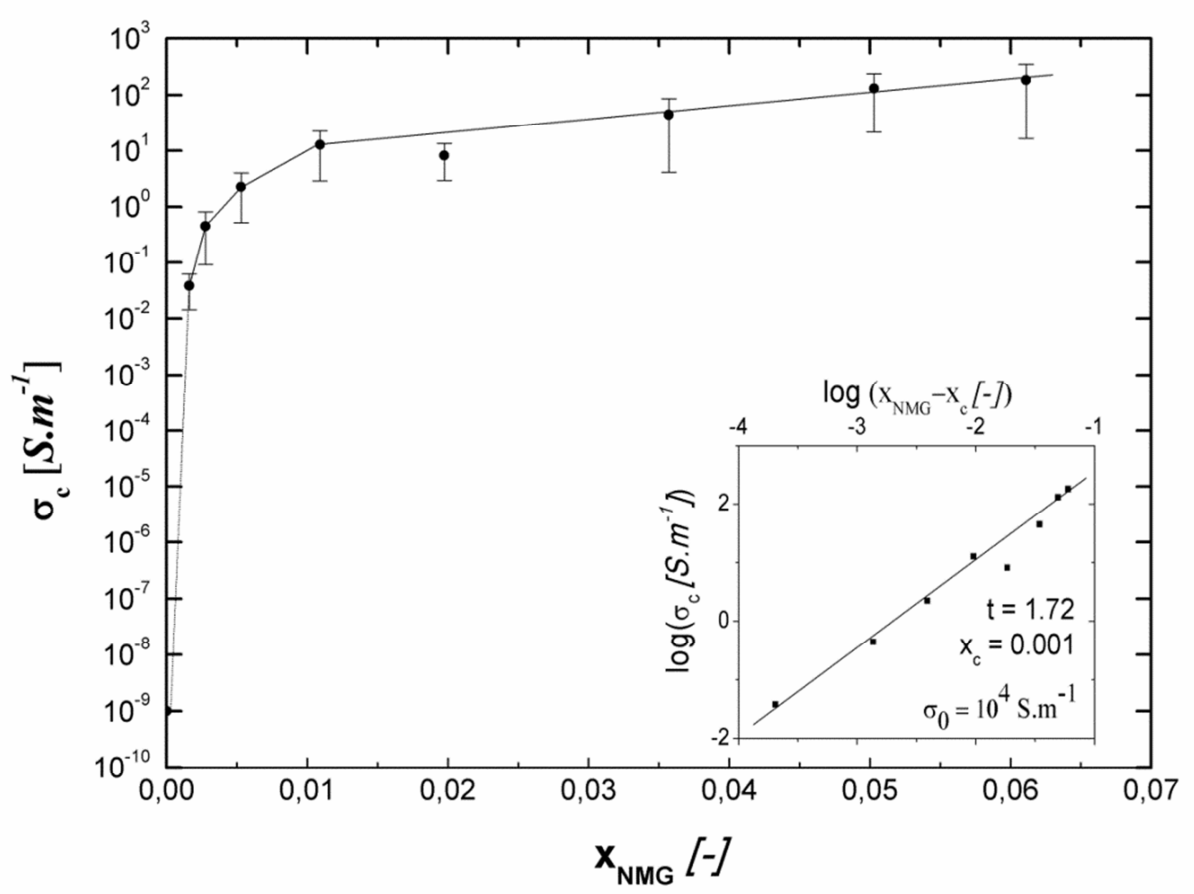

Figure 7: Measured electrical conductivity of the nanocomposite specimens. Inset: Log-Log plot illustrating the percolation model.

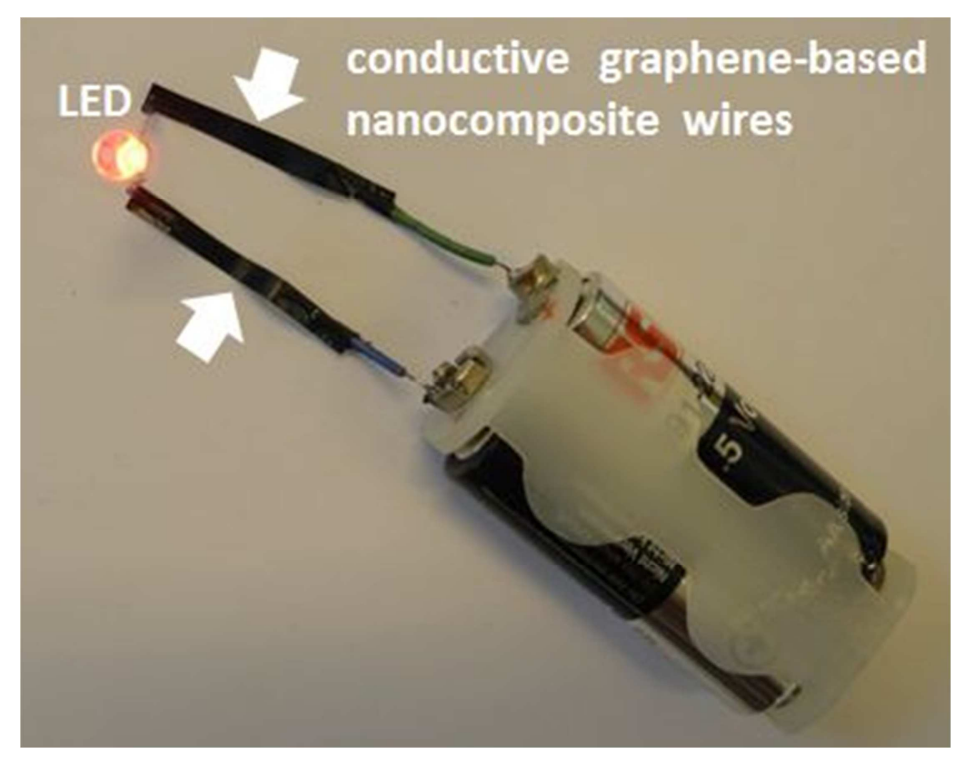

Figure 8: LED setup with conductive graphene-based nanocomposite wires (sample 8) in replacement of classical copper wires. 


\begin{tabular}{|c|c|c|c|c|c|c|c|c|c|}
\hline Sample reference & 0 & 1 & 2 & 3 & 4 & 5 & 6 & 7 & 8 \\
\hline$x N M G[-]$ & 0.0 & $1.6 \times 10^{-3}$ & $3.0 \times 10^{-3}$ & $5.0 \times 10^{-3}$ & $1.0 \times 10^{-2}$ & $2.0 \times 10^{-2}$ & $3.5 \times 10^{-2}$ & $5.0 \times 10^{-2}$ & $6.1 \times 10^{-2}$ \\
\hline
\end{tabular}

Table 1: NMG volume fraction $\left(x_{N M G}\right)$ for each NMG/latex suspension 